\title{
Osteoarticular Infection of the Shoulder Joint Due to Trichophyton Spp. in a Dog
}

\author{
Julien Alexandre Feline ${ }^{1}$ Julien Bernard Cabassu ${ }^{1}$ \\ ${ }^{1}$ Clinique Vétérinaire Cabassu, Marseille, France \\ VCOT Open 2021;4:e99-e103.
}

\begin{abstract}
Address for correspondence Julien Bernard Cabassu, DVM, DACVS DECVS, Clinique Vétérinaire Cabassu, 12 Avenue du Prado, 13006 Marseille, France (e-mail: juliencabassu@gmail.com).
\end{abstract}

\begin{abstract}
A 10-year-old Labrador Retriever was referred for persistent lameness due to chronic right shoulder pain, unresponsive to various pain management therapies. Radiographs indicated signs of severe degenerative changes in the joint. Synovial fluid analysis was not diagnostic. Septic arthritis was suspected based on computed tomography and clinical signs. Arthroscopy allowed joint exploration, tissue biopsies, and copious joint lavage. Trichophyton spp. proliferation was identified on antimicrobial culture and histological analysis on samples obtained during arthroscopy. Oral griseofulvin therapy was initiated. Two months later, the referring veterinarian decided to interrupt the treatment after a negative synovial culture despite persistent lameness. Euthanasia

Keywords

- septic arthritis

- dermatophytosis

- shoulder joint

- dog was elected upon after pain also appeared on the tarsus; a post-mortem exam was not authorized by the owner. The origin of the infection remains unclear as this patient had no skin lesions and its immunological status was unknown. However, dermatophytosis has been reported in healthy dogs without skin lesions. To the author's knowledge, this is the first report of an osteoarticular infection with a dermatophyte in a dog.
\end{abstract}

\section{Introduction}

Osteomyelitis is an inflammatory process usually secondary to infection which involves the bone and its medulla. Longterm infection leads to necrosis with lysis and new bone formation. ${ }^{1}$ The adjacent joints may be involved, and it may be difficult to assess whether the infectious arthritis or the adjacent osteomyelitis is the primary lesion.

Joint and bone infections are usually caused by bacteria inoculated either through penetrating wounds or hematogenous dissemination. ${ }^{2}$ Among the rare cases of mycotic osteoarthritic infections reported in dogs, fungi such as Blastomyces, Geomyces, Talaromyces, Coccidioides, Histoplasma, and Cryptococcus ssp. have been found. ${ }^{3-5}$

\section{Case History}

A 10-year-old Labrador Retriever was referred because of a 3month history of right thoracic limb lameness non-respond-

received

September 17, 2020

accepted after revision

August 5, 2021
DOI https://doi.org/

$10.1055 / \mathrm{s}-0041-1735842$.

ISSN 2625-2325. ing to various pain managements, including nonsteroidal anti-inflammatory drugs and corticosteroids. On presentation, non-weight bearing lameness with severe muscle atrophy of the right thoracic limb was observed. Vital signs were within normal limits. Extension and flexion of the right shoulder joint were painful, and the bicipital test was positive. Withdrawal reflex was intact on both front limbs. Postural reaction was not attempted due to the severe pain. No pain was elicited on the right elbow and carpus. An articular lesion of the scapulohumeral joint was highly suspected, although neurological involvement remained possible. Mediolateral radiographs of the right shoulder joint, taken by the referring veterinarian, revealed an illdefined punched-out osteolytic lesion at the articular surface of the caudal glenoid cavity and the humeral head with new bone formation at the margins of the aforementioned lysis (-Fig. 1).

Computed tomography (CT) revealed a large amount of rough and irregularly outlined new bone formation at the

\section{(c) 2021. The Author(s).}

This is an open access article published by Thieme under the terms of the Creative Commons Attribution License, permitting unrestricted use, distribution, and reproduction so long as the original work is properly cited. (https://creativecommons.org/licenses/by/4.0/)

Georg Thieme Verlag KG, Rüdigerstraße 14, 70469 Stuttgart, Germany 


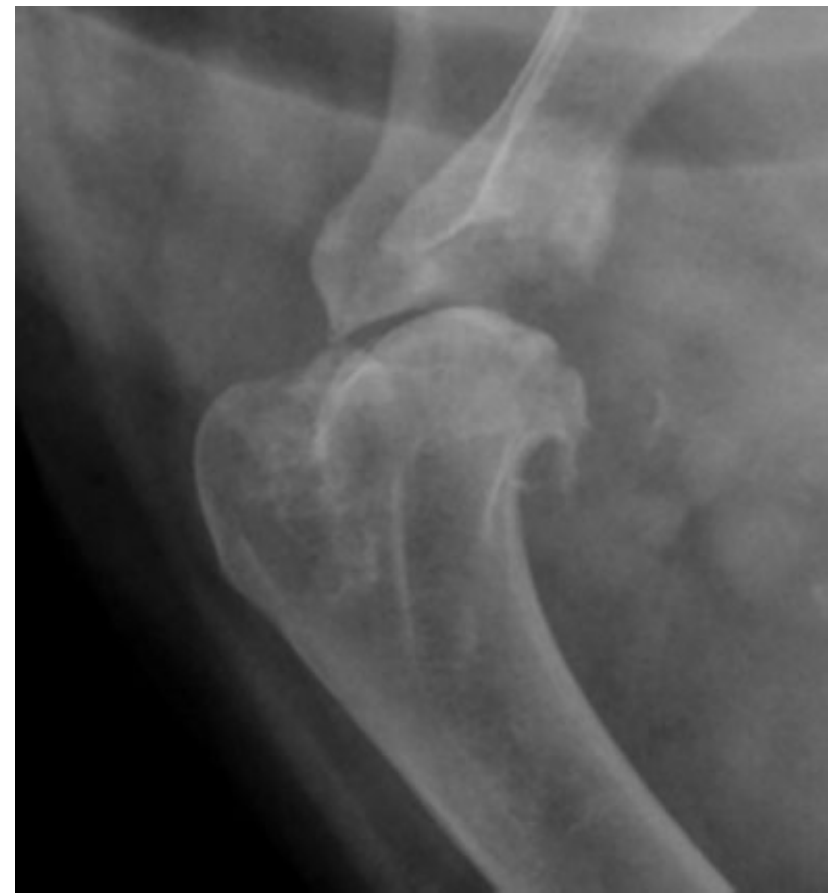

Fig. 1 Radiograph of the shoulder joint, mediolateral view. Ill-defined punched-out osteolysis and new bone formation at the articular surface of the caudal glenoid cavity and humeral head with adjacent subchondral bone sclerosis, with mineral opacity in the bicipital groove. The joint seemed distended.

caudal aspect of the humeral head, caudal border of the glenoid cavity, at the distal tip of the supraglenoid tubercle and along the bicipital groove ( $\mathbf{- F i g}$. 2A). The spontaneous attenuation of the joint effusion was high (20-40 Hounsfield unit, - Fig. 2B). In the caudal aspect of the humeral head, several small, round-shaped hypoattenuated defects were visible in the subchondral bone, surrounded by substantial sclerosis.

In conclusion, severe right scapulohumeral joint distension, consistent with active and marked inflammation, was observed. Furthermore, and concomitantly, signs compatible with osteochondritis dissecans (OCD) of the humeral head, chronic bicipital tendinopathy and osteoarthrosis were also observed. Based on clinical examination and the imaging findings, septic arthritis was highly suspected.

A synovial fluid sample was aspirated for cytology; however, due to blood contamination it was non-diagnostic. Haematologic exams and biochemical profile were unremarkable.

A shoulder joint arthroscopy via lateral approach was performed to explore and to obtain tissue samples (-Fig. 3). A focal defect of the cartilage and subchondral bone on the caudal humeral head was found, compatible with OCD ( $\boldsymbol{\text { Fig. }} \mathbf{3 A}$ ), as well as severe proliferative synovitis and eburnation on the glenoid cavity. The cranial compartment of the joint, including the bicipital tendon, could not be seen due to the severe synovial proliferations in this location. Samples of cartilage with subchondral bone from the caudal humeral head defect and synovial membrane were taken for histological analysis and microbiological culture with

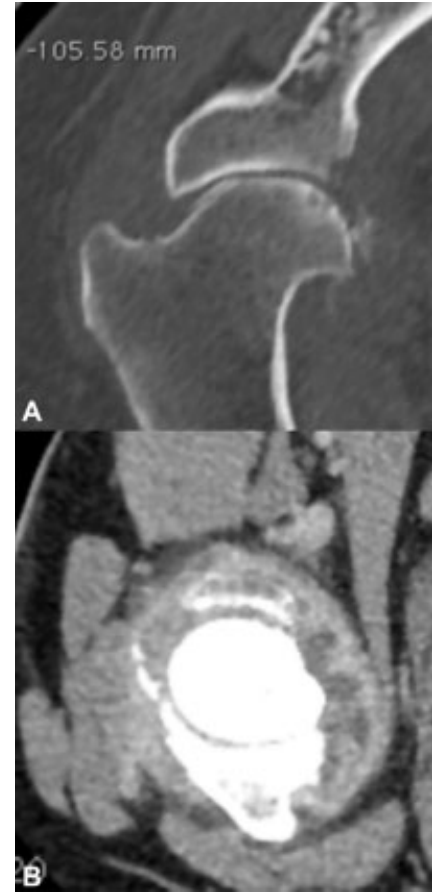

Fig. 2 Computed tomography of the right shoulder. (A) Large amount of rough and irregularly outlined new bone formation. In the caudal aspect of the joint, a large amorphous mineral area that was not in contact with any osseous structure was observed. A thin linear mineral flap is also observed in the cranial aspect of the joint. The articular surface within the entire joint was slightly irregularly outlined. (B) The joint was severely distended, and the joint capsule thickened and intensely enhanced following intravenous contrast administration.

antibiogram, respectively (-Fig. 3B). The joint was lavaged with 2L of sterile saline, before standard closure. The patient was discharged 24 hours postoperatively with standard antimicrobial therapy of amoxicillin/clavulanate

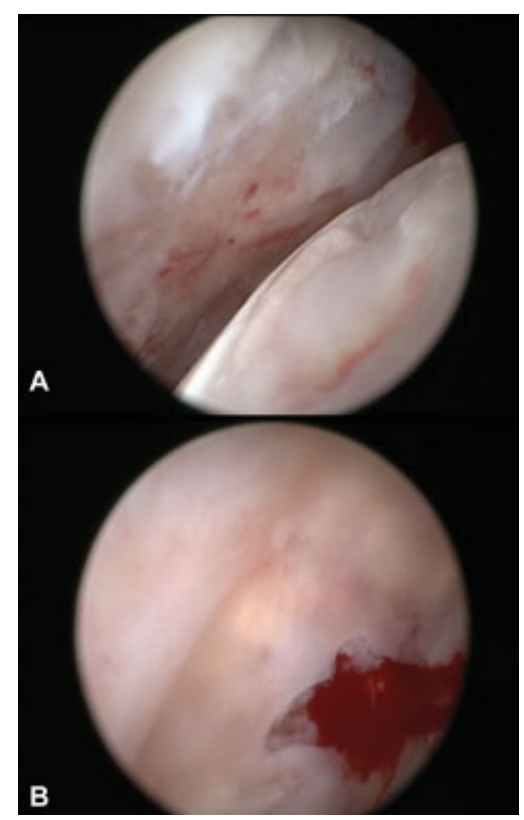

Fig. 3 Arthroscopy of the right shoulder. (A) Focal osteochondral defect on the caudal surface of the humeral head filled with fibrocartilaginous tissue. (B) Curettage of this lesion allowed tissues sampling. 


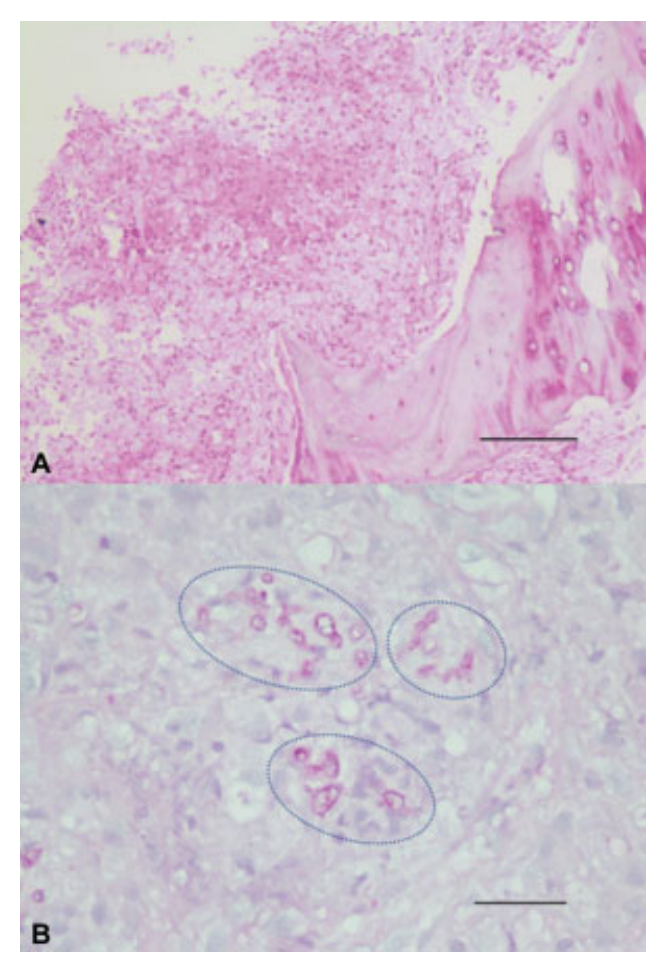

Fig. 4 Histology from tissues withdrawn during the arthroscopy. (A) $100 \times$, Haematoxylin and eosin stain, granulomatous infiltrate adjacent to a scalloped trabecula of bone; (B) $400 \times$, Periodic acid Schiff, intralesionnal fungal hyphae are visualized (dotted circles).

(12.5 mg/kg per os [p.o.] q12h, Kesium, Ceva, France), pending culture results. Pain was managed with meloxicam (0.1 mg/kg p.o. q24h, Meloxidyl, Ceva, France) and tramadol ( $3 \mathrm{mg} / \mathrm{kg}$ p.o. q12h, Tralieve, Dechra, United Kingdom) for 7 days.

Aerobic and anaerobic bacterial cultures were negative. Histologic analysis revealed synovitis and granulomatous osteomyelitis, with intra-macrophagic fungal components (-Fig. 4B), compatible with a deep osteoarticular mycosis. The microbiological culture was continued and at 4 weeks postoperatively, Trichophyton spp. was isolated.

Antimicrobial treatment was therefore changed to griseofulvin (10 mg/kg q12h, Fulviderm, Virbac, France) for 8 weeks. However, as no clinical improvement was observed, the referring veterinarian decided to interrupt the antifungal treatment, based on a negative synovial fluid culture obtained by joint aspiration.

Ipsilateral hindlimb lameness with tarsal joint effusion was observed 5 months after the initial diagnosis. Radiographs of the tarsus showed mild bone and joint surface remodelling. Although no synovial fluid was sampled, a septic extension to the tarsal joint was likely. Lameness and pain worsened and the general condition of the dog deteriorated rapidly and the owner elected euthanasia without consenting to a post-mortem examination.

\section{Discussion}

The most prevalent sylvatic dermatophyte that infects dogs is Trichophyton mentagrophytes which is usually spread to animals and humans by direct contact with contaminated hair, scales or by fomites. ${ }^{5}$ Most Trichophyton infections are suspected to be due to contact with infected rodents or their nests as they are more adapted to rodents and hedgehogs. 6,7 Immunosuppression and endocrine disorders are risk factors for dermatophytosis in pets, especially spontaneous or iatrogenic hyperadrenocorticism. ${ }^{6,8}$

In the reported case and in others, predisposing factors remained undefined. ${ }^{5}$ Unfortunately, several circumstances beyond our control resulted in the inability to further investigate and to explore pathogenetic modalities for this uncommon joint infection; that is, the remote and distant location the owner was living in, the serious financial constraints and the unwillingness to consent to further exams such as necropsy. Also, the immunological status of the animal remained ill-explored; although blood analyses were unremarkable, the possibility of hyperadrenocorticism had not been investigated. Immunological suppression secondary to steroids treatment seemed unlikely as it was prescribed for a short period of time ( 2 weeks) and at a low dose of $0.5 \mathrm{mg} / \mathrm{kg}$. Direct or penetrating inoculation into the joint is less likely, given the lack of any trauma history; nevertheless, contact with rodents and any previously undetected penetrating wound cannot be completely excluded. Thus, haematogenous spreading to the shoulder joint seems a possible explanation.

A prevalence of 19.79 to $49.1 \%$ of dermatophytosis has been reported in asymptomatic pets; however, geography, climate and lifestyle are important factors to consider. ${ }^{9,10}$ It is possible that the patient in our report was asymptomatically and chronically infected. No culture from the skin was performed to explore this hypothesis; however, no dermatological abnormality was seen during examination nor reported by the owner.

Deep infection such as septic arthritis or osteomyelitis secondary to Trichophyton's infection is extremely rare. ${ }^{6}$ One report in human medicine describes a Trichophyton's proliferation as a complication associated with surgery of a calcaneus fracture treated with external fixator. ${ }^{11}$ In our case, the dog had undergone an osteosynthesis on the contralateral tibia 6 years before. Radiographs from the surgical site were also performed at the time of diagnosis without radiographic signs of infection. In veterinary patients, there are few case reports describing uncommon fungal proliferation such as Oxyporus corticola or Rasamsonia piperina causing osteomyelitis, or Blastomyces and Talaromyces georgiensis causing arthritis. ${ }^{5,12-14}$ To the author's knowledge, this is the first report of Trichophyton's osteoarticular infection in a dog.

A septic arthritis was suspected on presentation based on the involvement of a single joint, specially a proximal joint, associated with severe pain and the severity of radiographic signs. $^{3}$ The following diagnostic plan was then elected, advanced imaging, cytology (contaminated with blood), and minimally invasive tissues biopsies and joint exploration, which allowed this diagnosis.

Computed tomography allowed a better assessment of the osteoarticular remodelling seen on radiographs. Images of 
the CT associated with clinical signs made septic arthritis more likely, even though a tumour remained a possible differential diagnosis.

In septic arthritis, the gold standard for diagnosis includes identification of the pathogen on a culture. Synovial fluid aspiration has only 30 to $50 \%$ likelihood to identify pathogens with standard culture medium and sensibility can be increased to 53 to $80 \%$ using blood enriched media, when considering bacterial infections. ${ }^{15-17}$ However, by adding synovial membrane (to synovial fluid) in the culture, it has been observed that the efficacity to isolate the causal pathogen could reach $100 \%{ }^{18}$ In our case, culture on synovial aspiration was not attempted as it was already planned to sample tissues during arthroscopy. It could have been interesting to compare results from synovial fluid only and samples harvested during arthroscopy. This case also illustrates the importance to complete a culture for both bacterial and fungal culture when a septic arthritis is suspected, assuming that it can take a longer time to grow fungi than bacteria. Here, the first results regarding aerobic and anaerobic bacteria were negative but Trichophyton spp. was isolated 10 days later.

In humans, arthroscopic management of septic arthritis is the preferred technique for most, especially large joints. ${ }^{19-21}$ In our case, we elected to perform arthroscopy first, because cytology alone was not diagnostic and tissue samples from obvious affected area were needed for diagnostic purpose. ${ }^{18}$ Second, copious joint lavage is known to decrease pain associated with joint effusion and inflammation, and is highly recommended in a patient with septic arthritis. ${ }^{3}$ Moreover, joint exploration allowed the visualization of a former OCD lesion seen on CT; exposure of the subchondral bone of the glenoid cavity was visible and hard on palpation. A kissing lesion was suspected. Based on the symmetry of the lesion on the CT and the quality of the sample harvested we did not attempt to sample the glenoid cavity. This OCD lesion could have been the primary lesion leading to (osteo)arthritis and thus could be seen as locus minoris resistentiae for secondary infection.

Griseofulvin, a fungistatic antibiotic medication primarily used in dermatophytic infections, was empirically elected. ${ }^{22}$ As no improvement was achieved, its effectiveness in this case remained uncertain and a possible resistance must be questioned. Mean duration of treatment in small animals seems to be close to 40 days which means it could take longer to eliminate Trichophyton spp., and prolonged treatment may have been successful. ${ }^{23}$ Based on a recent consensus itraconazole, a fungistatic triazole, seems to be a more efficient treatment in Trichophytosis and could have been the preferred treatment in this case. ${ }^{7,22}$ In the unique similar report in human medicine, complete recovery was achieved after a 3 months' treatment with itraconazole. ${ }^{11}$

\section{Conclusion}

In a dog suffering from severe pain in the shoulder, synovial membrane as well as subchondral bone biopsies from representative areas were harvested arthroscopically and allowed diagnosis of a dermatophytic septic arthritis and osteomyelitis due to Trichophyton spp.

Oral griseofulvin for 2 months was an unsuccessful treatment. Itraconazole has been efficient to treat a similar osteomyelitis in a human. Origin of the infection is unclear, although dermatophytosis has been reported in healthy animals and can lead to the suspicion of a haematogenous spread.

\section{Conflict of Interest Statements}

None of the authors of this article has a financial or personal relationship with other people or organizations that could inappropriately influence or bias the content of the paper.

\section{Acknowledgments}

The authors would like to thank Caroline Laprie, DVM, DECVP, for her assistance in the interpretation of the histological analysis and descriptions of illustrations.

\section{References}

1 Birt MC, Anderson DW, Bruce Toby E, Wang J. Osteomyelitis: recent advances in pathophysiology and therapeutic strategies. J Orthop 2016;14(01):45-52

2 Nelson RW, Couto CG. Small Animal Internal Medicine. St. Louis, Missouri: Elsevier Health Sciences; 2014

3 Ettinger SJ, Feldman EC, Cote E. Textbook of Veterinary Internal Medicine. St. Louis, Missouri: Elsevier Health Sciences; 2017

4 Erne JB, Walker MC, Strik N, Alleman AR. Systemic infection with Geomyces organisms in a dog with lytic bone lesions. J Am Vet Med Assoc 2007;230(04):537-540

5 Okada K, Kano R, Hasegawa T, Kagawa Y. Granulomatous polyarthritis caused by Talaromyces georgiensis in a dog. J Vet Diagn Invest 2020;32(06):912-917

6 Sykes JE. Canine and Feline Infectious Diseases. London, UK: Elsevier Health Sciences; 2013

7 Moriello KA, Coyner K, Paterson S, Mignon BClinical Consensus Guidelines of the World Association for Veterinary Dermatology. Diagnosis and treatment of dermatophytosis in dogs and cats. Vet Dermatol 2017;28(03):266-e68

8 Greene CE. Infectious Diseases of the Dog and Cat. St. Louis, Missouri: Elsevier Health Sciences; 2013

9 Debnath C, Mitra T, Kumar A, Samanta I. Detection of dermatophytes in healthy companion dogs and cats in eastern India. Majallah-i Tahqiqat-i Dampizishki-i Iran 2016;17(01): 20-24

10 Zdovc I, Mičunovi J, Pirš T, Ocepek M. P-11 Occurrence of dermatophytes in dogs and cats and their susceptibility to antifungal drugs. Vet Dermatol 2004;15:44

11 Waryasz GR, Bariteau JT. Trichophyton rubrum osteomyelitis after calcaneus external fixation pin stabilization of a pilon fracture. J Foot Ankle Surg 2014;53(04):480-484

12 Brockus CW, Myers RK, Crandell JM, Sutton DA, Wickes BL, Nakasone KK. Disseminated Oxyporus corticola infection in a German shepherd dog. Med Mycol 2009;47(08):862-868

13 Lodzinska J, Cazzini P, Taylor CS, et al. Systemic Rasamsonia piperina infection in a German shepherd cross dog. JMM Case Rep 2017;4(10):e005125

14 Woods KS, Barry M, Richardson D. Carpal intra-articular blastomycosis in a Labrador retriever. Can Vet J 2013;54(02):167-170

15 Scharf VF, Lewis ST, Wellehan JF, et al. Retrospective evaluation of the efficacy of isolating bacteria from synovial fluid in dogs with suspected septic arthritis. Aust Vet J 2015;93(06):200-203 
16 Clements DN, Owen MR, Mosley JR, Carmichael S, Taylor DJ, Bennett D. Retrospective study of bacterial infective arthritis in 31 dogs. J Small Anim Pract 2005;46(04):171-176

17 Marchevsky AM, Read RA. Bacterial septic arthritis in 19 dogs. Aust Vet J 1999;77(04):233-237

18 Bennett D, Taylor D. Bacterial infective arthritis in the dog. J Small Anim Pract 1988;29(04):207-230

19 Balabaud L, Gaudias J, Boeri C, Jenny JY, Kehr P. Results of treatment of septic knee arthritis: a retrospective series of 40 cases. Knee Surg Sports Traumatol Arthrosc 2007;15(04): 387-392
20 El-Sayed AMM. Treatment of early septic arthritis of the hip in children: comparison of results of open arthrotomy versus arthroscopic drainage. J Child Orthop 2008;2(03):229-237

21 Nusem I, Jabur MK, Playford EG. Arthroscopic treatment of septic arthritis of the hip. Arthroscopy 2006;22(08):902.e1-902.e3

22 Plumb DC. Plumb's Veterinary Drug Handbook. 9th edition. Hoboken, NJ: John Wiley \& Sons; 2018

23 Balda AC, Otsuka M, Gambale W, Larsson CE. P-12 Comparative study of griseofulvin and terbinafine therapy in the treatment of canine and feline dermatophytosis. Vet Dermatol 2004;15:44 